\title{
Macrophage ABCA1 and ABCG1, but not SR-BI, promote macrophage reverse cholesterol transport in vivo
}

\author{
Xun Wang, ${ }^{1}$ Heidi L. Collins, ${ }^{2}$ Mollie Ranalletta, ${ }^{3}$ Ilia V. Fuki, ${ }^{1}$ Jeffrey T. Billheimer, ${ }^{1}$ \\ George H. Rothblat, ${ }^{2}$ Alan R. Tall, ${ }^{3}$ and Daniel J. Rader ${ }^{1}$ \\ 1Institute for Translational Medicine and Therapeutics and Cardiovascular Institute, School of Medicine, University of Pennsylvania, Philadelphia, \\ Pennsylvania, USA. ${ }^{2 T h e}$ Children's Hospital of Philadelphia, Philadelphia, Pennsylvania, USA. ${ }^{3}$ Division of Molecular Medicine, \\ Department of Medicine, Columbia University College of Physicians and Surgeons, New York, New York, USA.
}

\begin{abstract}
Macrophage ATP-binding cassette transporter A1 (ABCA1), scavenger receptor class B type I (SR-BI), and ABCG1 have been shown to promote cholesterol efflux to extracellular acceptors in vitro and influence atherosclerosis in mice, but their roles in mediating reverse cholesterol transport (RCT) from macrophages in vivo are unknown. Using an assay of macrophage RCT in mice, we found that primary macrophages lacking ABCA1 had a significant reduction in macrophage RCT in vivo, demonstrating the importance of ABCA1 in promoting macrophage RCT, however substantial residual RCT exists in the absence of macrophage ABCA1. Using primary macrophages deficient in SR-BI expression, we found that macrophage SR-BI, which was shown to promote cholesterol efflux in vitro, does not contribute to macrophage RCT in vivo. To investigate whether macrophage ABCG1 is involved in macrophage RCT in vivo, we used ABCG1-overexpressing, -knockdown, and -knockout macrophages. We show that increased macrophage ABCG1 expression significantly promoted while knockdown or knockout of macrophage ABCG1 expression significantly reduced macrophage RCT in vivo. Finally, we show that there was a greater decrease in macrophage RCT from cells where both ABCA1 and ABCG1 expression were knocked down than from ABCG1-knockdown cells. These results demonstrate that $A B C A 1$ and $A B C G 1$, but not SR-BI, promote macrophage RCT in vivo and are additive in their effects.
\end{abstract}

\section{Introduction}

Reverse cholesterol transport (RCT) is believed to be the primary mechanism by which HDL and its major protein apoA-I protect against atherosclerosis (1). While all cells are capable of effluxing cholesterol, cholesterol efflux from macrophages is the most important with regard to atherosclerosis and represents the first critical step of RCT (2). Several molecules have been suggested to play important roles in determining macrophage cholesterol efflux. ATP-binding cassette transporter A1 (ABCA1) mediates cholesterol efflux from macrophages to lipid-free apoA-I (3-5). Macrophages lacking ABCA1 have reduced cholesterol efflux to lipid-free apoA-I in vitro $(6,7)$. Furthermore, mice that lack ABCA1 expression in macrophages developed accelerated atherosclerosis (7). Conversely, mice with macrophage overexpression of ABCA1 have decreased atherosclerosis (8). However, it has not been demonstrated to what extent $\mathrm{ABCA} 1$ is critical for macrophage cholesterol efflux and RCT in vivo.

Scavenger receptor class B type I (SR-BI) is expressed in hepatocytes and macrophages and mediates cholesterol efflux to mature HDL in vitro (9). Hepatic expression of SR-BI promot-

Nonstandard abbreviations used: ABCA1, ATP-binding cassette transporter A1; acLDL, acetylated LDL; BMM, BM-derived macrophage; DKD, double-KD; KD, knockdown; LDLR, LDL receptor; LXR, liver X receptor; MPM, mouse peritoneal macrophage; OE, overexpressing; RCT, reverse cholesterol transport; SR-BI, scavenger receptor class $B$ type $I$.

Conflict of interest: G.H. Rothblat has consulted for Pfizer; A.R. Tall has consulted for Amira Pharmaceuticals, Bristol-Myers Squibb, Merck, and Pfizer; and D.J. Rader has consulted for Bristol-Myers Squibb, Lilly, Merck, Merck/Schering-Plough, Novartis, Pfizer, and Wyeth on topics related to reverse cholesterol transport.

Citation for this article: J. Clin. Invest. 117:2216-2224 (2007). doi:10.1172/JCI32057. ed liver HDL-cholesterol uptake and increased RCT in vivo (10). Mice with SR-BI-deficient macrophages have increased atherosclerosis $(11,12)$. However, it remains unclear whether the macrophage SR-BI can promote net macrophage cholesterol efflux and RCT in vivo.

ABCG1 was recently identified as being a mediator of macrophage cholesterol efflux to mature HDL in vitro. Macrophages lacking ABCG1 expression have impaired cholesterol efflux to HDL but not to lipid-free apoA-I in vitro $(13,14)$. ABCA1 and ABCG1 have been shown to function cooperatively to remove cholesterol from cells in vitro (15). Mice lacking ABCG1 have evidence of lipid accumulation in certain tissue macrophages $(14,16)$, but the mechanism of this has not been proven to be impaired cholesterol efflux and RCT in vivo. Furthermore, the role of macrophage ABCG1 in the development of atherosclerosis remains uncertain: independent studies have shown that mice that lack macrophage ABCG1 expression had either decreased $(17,18)$ or increased $(19)$ atherosclerosis. Therefore, it is critical to establish whether ABCG1 contributes to macrophage cholesterol efflux and RCT in vivo.

In the present study, we quantitatively assessed the roles of macrophage ABCA1, SR-BI, or ABCG1 in macrophage RCT in vivo. Primary macrophages lacking ABCA1 demonstrated a significant, $30 \%$ reduction in macrophage $\mathrm{RCT}$, indicating substantial residual efflux in vivo. Primary macrophages lacking SR-BI did not differ from WT macrophages in macrophage RCT, indicating little role for macrophage SR-BI in this process in vivo. Finally, using J774 macrophages with either increased or reduced ABCG1 expression as well as primary macrophages lacking ABCG1 expression, we determined that ABCG1 played a critical role in promoting macrophage RCT in vivo. Furthermore, by knocking down ABCA1 and 
A

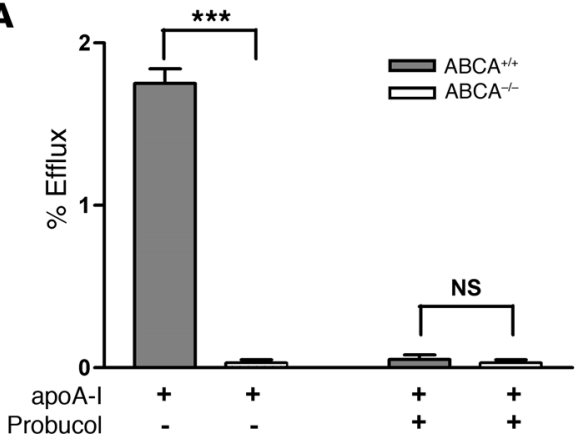

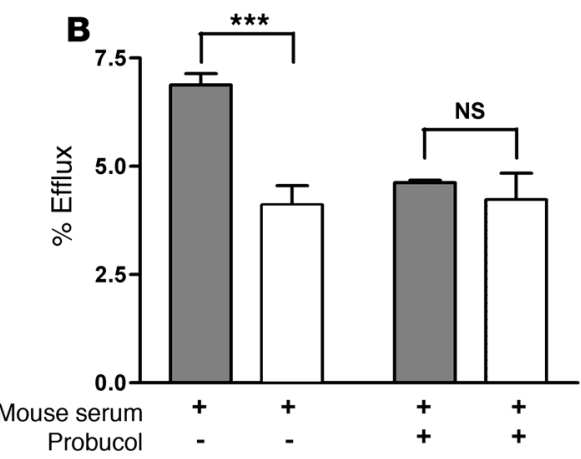

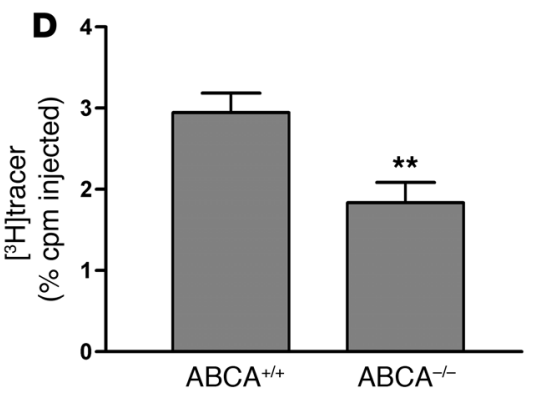

Figure 1

ABCA1-deficient BMMs have reduced cholesterol efflux ex vivo and RCT in vivo. (A) BMMs from WT $\left(\mathrm{ABCA} 1^{+/+}\right)$or $\mathrm{ABCA} 1-\mathrm{KO}\left(\mathrm{ABCA} 1^{-/-}\right)$mice were labeled with $\left[{ }^{3} \mathrm{H}\right]$ cholesterol and loaded with $25 \mu \mathrm{g} / \mathrm{ml}$ acLDL for 24 hours. Cells were equilibrated for 18 hours in the presence of $1 \mu \mathrm{M}$ LXR agonist (GW3965). Then, cells were incubated for 2 hours in either the presence of absence of probucol $(20 \mu \mathrm{M})$. Cholesterol efflux was determined in the presence of lipid-free apoA-I $(10 \mu \mathrm{g} / \mathrm{ml})$ for 2 hours. Data are expressed as mean \pm SD; $n=3 .{ }^{* \star *} P<0.001$. (B) Cholesterol efflux was determined as described above, except $2.5 \%$ mouse whole serum was used as the acceptor. Data are expressed as mean $\pm \mathrm{SD} ; n=3 .{ }^{* * *} P<0.001$. (C and D) For the in vivo RCT experiment, WT C57BL/6 mice that had been treated with an LXR agonist for 2 weeks were injected i.p. with $\left[{ }^{3} \mathrm{H}\right]$ cholesterol-labeled, acLDL-loaded, and $\mathrm{LXR}$ agonist-treated $\mathrm{BMMs}$ from $\mathrm{ABCA} 1^{+/+}$or $\mathrm{ABCA} 1^{-/-}$mice. Mice were bled at $2,6,24$, and 48 hours after injection. $n=8$ mice per group. Data are expressed as the percentage of tracer relative to total $\mathrm{cpm}$ tracer injected $\pm \mathrm{SEM}$. ${ }^{* *} P<0.01$. Results are representative of 2 independent experiments. (C) Time course of $\left[{ }^{3} \mathrm{H}\right]$ cholesterol distribution in plasma. Individual time points and areas under the curve were determined and compared. (D) Fecal $\left[{ }^{3} \mathrm{H}\right]$ tracer levels. Feces were collected continuously from 0 to 48 hours.

ABCG1 simultaneously, we demonstrated that ABCA1 and ABCG1 functioned cooperatively to mediate macrophage RCT in vivo.

\section{Results}

ABCA1-deficient macrophages exhibit reduced RCT in vivo. To investigate whether macrophage expression of ABCA1 is required for RCT in vivo, we first characterized cholesterol efflux from BM-derived macrophages (BMMs) of ABCA1-KO mice to either apoA-I or mouse whole serum. Ablation of ABCA1 expression in macrophages completely inhibited cholesterol efflux to lipid-free apoA-I (Figure 1A). More importantly, cholesterol efflux from ABCA1-KO BMMs to mouse whole serum was decreased by $40 \%$ compared with that from WT BMMs (Figure 1B). To confirm that the reduced cholesterol efflux to apoA-I and mouse whole serum was the result of ABCA1 deficiency, we used probucol to specifically inhibit the ABCA1-mediated cholesterol efflux in WT macrophages. After the addition of probucol, cholesterol efflux from WT BMMs was reduced to the level of efflux from ABCA1-KO BMMs (Figure 1, A and B).
Next, we investigated the role of macrophage expression of ABCA1 in $\mathrm{RCT}$ in vivo. Cholesterol-loaded, liver $X$ receptor (LXR) agonist-treated, and $\left[{ }^{3} \mathrm{H}\right]$ cholesterol-labeled ABCA1-KO and WT BMMs were injected i.p. into WT C57BL/6 mice that had been treated with an LXR agonist for 2 weeks. We then followed the $\left[{ }^{3} \mathrm{H}\right]$ tracer level in plasma at 2, 6, 24, and 48 hours after injection and integrated the $\left[{ }^{3} \mathrm{H}\right]$ tracer level in feces collected through the 0 - to 48 -hour period. Plasma $\left[{ }^{3} \mathrm{H}\right]$ tracer levels from mice injected with ABCA1-KO BMMs were decreased by $27 \%(P<0.01)$, as calculated by the area under the curve after injection of labeled macrophages (Figure 1C). Furthermore, fecal $\left[{ }^{3} \mathrm{H}\right]$ tracer excretion from ABCA1-KO $\mathrm{BMM}$-injected mice compared with control BMM-injected mice was reduced by $37 \%(P<0.01$; Figure $1 \mathrm{D})$. An additional independent experiment using ABCA1-KO and WT BMMs yielded similar results (data not shown). Furthermore, 2 independent RCT experiments using mouse peritoneal macrophages (MPMs) from ABCA1-deficient and control mice resulted in an average decrease of $38 \%(P<0.001)$ in plasma $\left[{ }^{3} \mathrm{H}\right]$ tracer level as calculated by the area under curve and a $25 \%$ decrease $(P<0.05)$ in fecal $\left[{ }^{3} \mathrm{H}\right]$ tracer level (Supplemental Figure 1, A and B; supplemental material available online with this article; doi:10.1172/JCI32057DS1). These data indicate that macrophage expression of ABCA1 plays an important role in RCT from macrophages in vivo but that substantial efflux and RCT still occur even in the absence of macrophage ABCA1.

SR-BI-deficient macrophages exhibit no significant reduction in RCT in vivo. The fact that substantial RCT took place even from ABCA1$\mathrm{KO}$ cells suggested the existence of alternative pathways by which cholesterol from macrophages can be effluxed in vivo. SR-BI, a receptor known to be expressed on the surface of macrophages, has been shown to mediate cholesterol efflux to HDL in vitro (9). Therefore, we investigated whether deletion of macrophage SR-BI reduced macrophage RCT in vivo. Consistent with a previous report (20), we found that cholesterol loading significantly reduced SR-BI protein expression in primary murine macrophages (Figure 2A). Therefore, we decided to use unloaded macrophages for the SR-BI experiments. First, we performed cholesterol efflux studies using BMMs isolated from SR-BI-KO and control mice. There was no difference in cholesterol efflux to either $\mathrm{HDL}_{3}$ or mouse whole serum from $\mathrm{SR}-\mathrm{BI}^{-/}$and control macrophages (Figure $2 \mathrm{~B}$ ). Next, we injected $\left[{ }^{3} \mathrm{H}\right]$ cholesterol-labeled $\mathrm{SR}-\mathrm{BI}^{-/-}$or control BMMs into WT mice to measure the rate of RCT in vivo. 
A

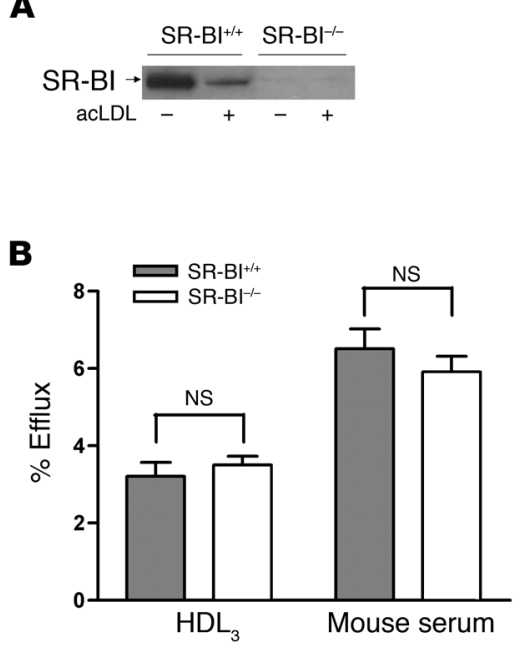

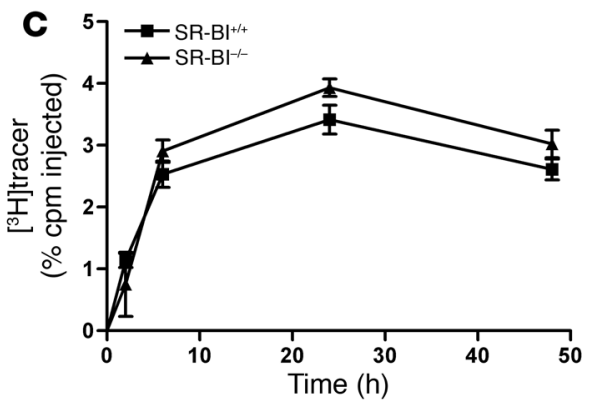

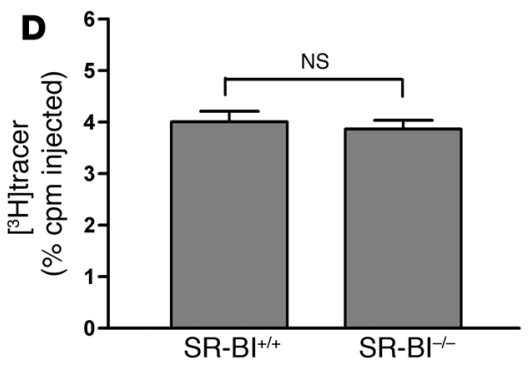

Figure 2

SR-BI-deficient BMMs have normal cholesterol efflux ex vivo and RCT in vivo. (A) Western blotting demonstrating the absence of SR-BI protein expression in SR-BI-/- macrophages. SR-BI was detected by Western blotting with anti-SR-BI antibody. Equal amounts of total proteins were loaded. (B) Mouse BMMs from WT $\left(\mathrm{SR}-\mathrm{BI}^{+/+}\right)$and SR-BI ${ }^{-/-}$mice were labeled with $\left[{ }^{3} \mathrm{H}\right]$ cholesterol for 24 hours. After equilibration in $0.2 \%$ BSA overnight, macrophages were incubated with either $25 \mu \mathrm{g} / \mathrm{ml} \mathrm{HDL}_{3}$ or $2.5 \%$ mouse whole serum for 2 hours. Values are mean $\pm \mathrm{SD} ; n=3$. Results are representative of 2 independent experiments. (C and D) The RCT experiment was performed as described in Figure 1 for $\mathrm{SR}-\left.\mathrm{BI}\right|^{+/+}$and $\mathrm{SR}-\mathrm{BI}^{-/-}$ BMMs, except that cells were not loaded with acLDL and neither cells nor mice were treated with GW3965. $n=6$ mice per group. Data are expressed as the percentage of tracer relative to total cpm tracer injected \pm SEM. Results are representative of 2 independent experiments. (C) Time course of $\left[{ }^{3} \mathrm{H}\right]$ cholesterol distribution in plasma. (D) Fecal $\left[{ }^{3} \mathrm{H}\right]$ tracer levels. Feces were collected continuously from 0 to 48 hours.

The $\left[{ }^{3} \mathrm{H}\right]$ tracer level in plasma at all time points measured and in feces collected over 48 hours were not significantly different in the 2 groups of mice (Figure 2, C and D). An independent RCT experiment using unloaded MPMs from control and $\mathrm{SR}-\mathrm{BI}^{-/-}$mice yielded similar results (Supplemental Figure 2, A and B), as did an experiment using cholesterol-loaded MPMs from control and $\mathrm{SR}-\mathrm{BI}^{-/-}$mice (data not shown). These data indicate that macrophage SR-BI does not contribute to macrophage RCT in vivo to any great extent and is unlikely to account for the residual RCT we observed from $\mathrm{ABCA} 1^{-/-}$macrophages.

Overexpression of ABCG1 in 7774 macrophages promotes cholesterol efflux ex vivo and RCT in vivo. ABCG1, another ATP-binding cassette family member, promotes cholesterol efflux to mature HDL particles and, like ABCA1, is upregulated by LXR (21). We investigated whether ABCG1 can mediate macrophage RCT in vivo. First, to determine whether overexpression of ABCG1 in macrophages can promote cholesterol efflux and RCT, we generated J774 macrophages stably overexpressing ABCG1 (J774-ABCG1-OE macrophages) by transducing WT J774 macrophages with lentivirus expressing the full-length ABCG1 cDNA. ABCG1 mRNA levels were significantly higher in J774-ABCG1-OE cells compared with control cells (Figure 3A), and Western blotting demonstrated that ABCG1 protein expression was greater in J774-ABCG1-OE macrophages than in control cells, in both the absence and presence of an LXR agonist
(Figure 3B). Furthermore, we observed a 40\% increase in cholesterol efflux to $\mathrm{HDL}_{3}$ and a $28 \%$ increase in cholesterol efflux to mouse whole serum from J774-ABCG1-OE macrophages loaded with acetylated LDL (acLDL) and treated with an LXR agonist (Figure 3C).

We assessed in vivo macrophage RCT by injecting $\left[{ }^{3} \mathrm{H}\right]$ cholesterol-labeled, cholesterol-loaded, and LXR agonist-treated J774ABCG1-OE and control J774 macrophages into LXR agonist-treated C57BL/6 WT mice. Plasma $\left[{ }^{3} \mathrm{H}\right]$ tracer levels in mice injected with J774 macrophages overexpressing ABCG1 were increased by $30 \%(P<0.01)$ as calculated by comparing the area under the curve for mice injected with J774-ABCG1-OE macrophages with that for mice injected with control macrophages (Figure 3D). Moreover, the fecal $\left[{ }^{3} \mathrm{H}\right]$ tracer excretion was $52 \%$ higher from mice injected with $\mathrm{J} 774$ macrophages overexpressing ABCG1 $(P<0.01$; Figure $3 \mathrm{E})$. These data indicate that elevated levels of macrophage ABCG1 promote macrophage RCT in vivo.

Silencing of ABCG1 in 7774 cells significantly reduces cholesterol efflux in vitro and $R C T$ in vivo. We generated lentiviral vectors encoding shRNA targeting murine ABCG1 and used them to transduce WT J774 cells in order to produce cell lines that have stably knocked down ABCG1 expression (J774-ABCG1-KD). J774 cells transduced with a lentiviral vector encoding shRNA targeting lacZ and otherwise passaged identically were used as the control cells (J774-control). Murine Abcg1 mRNA was reduced more than $50 \%$ in the untreated J774-ABCG1-KD cells and by 70\% in LXR agonist-treated J774-ABCG1-KD cells compared with similarly treated control cells (Figure 4A). ABCG1 protein levels were also substantially reduced in J774-ABCG1-KD cells (Figure 4B). ABCA1 and SR-BI expression did not differ in control and ABCG1$\mathrm{KD}$ cells under the various conditions studied (data not shown).

We investigated whether decreased expression of ABCG1 in J774 cells would cause reduced cholesterol efflux. After cells were cholesterol loaded with acLDL, cholesterol efflux to $\mathrm{HDL}_{3}$ was $20 \%$ less from J774-ABCG1-KD cells compared with control cells, whereas after cells were both cholesterol loaded and LXR agonist treated, cholesterol efflux to $\mathrm{HDL}_{3}$ was $35 \%$ lower from J774ABCG1-KD cells compared with control cells (Figure 4C). J774ABCG1-KD cells also had significantly reduced cholesterol efflux to whole mouse serum (Figure 4D).

To determine whether the reduced expression of ABCG1 influenced RCT in vivo, we injected $\left[{ }^{3} \mathrm{H}\right]$ cholesterol-labeled, cholesterol-loaded, and LXR agonist-treated J774-ABCG1-KD or control cells into LXR agonist-treated WT C57BL/ 6 mice. The plasma $\left[{ }^{3} \mathrm{H}\right]$ cholesterol levels in mice injected with J774-ABCG1-KD cells were $30 \%(P<0.001)$ lower than those in mice injected with J774 control cells as calculated by the area under curve (Figure 4E). Furthermore, the total fecal $\left[{ }^{3} \mathrm{H}\right]$ steroid excretion from mice injected with J774-ABCG1-KD cells was $40 \%$ lower than that from mice injected with control cells $(P<0.05$; Figure 4F), indicating that 

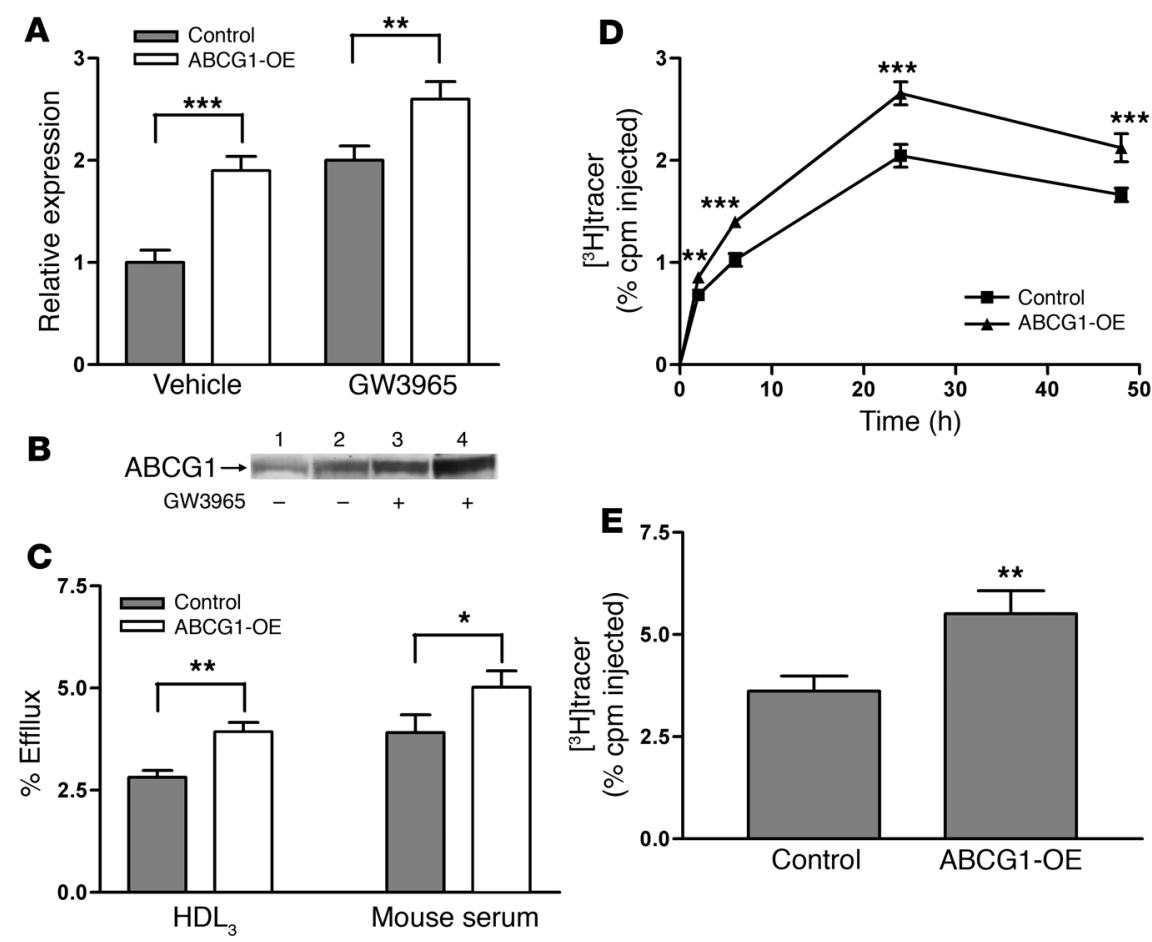

Figure 3

Overexpression of ABCG1 in $\mathrm{J} 774$ macrophages promotes cholesterol efflux in vitro and RCT in vivo. (A) Quantitative analysis of mRNA expression of abcg1 in ABCG1-OE and control cells by quantitative RT-PCR. Total RNA was extracted from cells that were treated with either vehicle or GW3965 $(1 \mu \mathrm{M})$ for 24 hours. Data are expressed as fold change \pm SD and normalized to mouse 18S rRNA. ${ }^{* *} P<0.01 ;{ }^{* * *} P<0.001$. (B) Western blot demonstrating the overexpression of $A B C G 1$. Control and ABCG1-OE cells were grown in either the absence or presence of GW3965 as indicated. ABCG1 was detected by Western blotting with anti-ABCG1 antibody. Equal amount of total proteins were loaded. Lanes 1 and 3 represent control cells. Lanes 2 and 4 represent ABCG1-OE cells. (C) Cholesterol efflux assay was performed as described in Figure 1. Cholesterol efflux was determined in the presence of $\mathrm{HDL}_{3}(25 \mu \mathrm{g} / \mathrm{ml})$ or $2.5 \%$ mouse whole serum for 4 hours. Data are expressed as mean \pm SD; $n=3 .{ }^{\star} P<0.05$; ${ }^{* *} P<0.01$. (D and E) The RCT experiment was done as described in Figure 1 with $\left[{ }^{3} \mathrm{H}\right]$ cholesterol-labeled, acLDLloaded, and LXR agonist-treated J774 control macrophages and ABCG1-OE macrophages. $n=8$ mice per group. Data are expressed as the percentage of tracer relative to total $\mathrm{cpm}$ tracer injected \pm SEM. ${ }^{* *} P<0.01 ;{ }^{* \star *} P<0.001$. (D) Time course of $\left[{ }^{3} \mathrm{H}\right]$ cholesterol distribution in plasma. Individual time points and areas under the curve were determined and compared. (E) Fecal $\left[{ }^{3} \mathrm{H}\right]$ tracer levels. Feces were collected continuously from 0 to 48 hours.

reduced expression of ABCG1 impairs macrophage RCT in vivo. Similar results were obtained in 2 additional independent experiments (data not shown).

Ablation of ABCG1 expression in primary macrophages significantly reduces $R C T$ in vivo. To confirm that $\mathrm{ABCG} 1$ can promote cholesterol efflux and RCT in vivo in primary macrophages, we used BMMs from ABCG1-KO mice. BMMs lacking ABCG1 had significantly decreased cholesterol efflux to $\mathrm{HDL}_{3}$ when cells were loaded with acLDL and treated with an LXR agonist (Figure 5A), whereas there was no difference in cholesterol efflux from ABCG1-KO BMMs to free apoA-I. When mouse whole serum was used as the cholesterol acceptor, cholesterol efflux was decreased 15\% from ABCG1-KD BMMs compared with WT control BMMs (Figure 5B). To test whether the residual cholesterol efflux from ABCG1-KO BMMs to mouse serum was ABCA1 mediated, we used probucol to specifically inhibit ABCA1-mediated cholesterol efflux and found that cholesterol efflux was reduced further, by $40 \%$ (Figure 5B). Overall, in cells lacking ABCG1 and treated with probucol to block ABCA1, cholesterol efflux was decreased by $70 \%$, suggesting that ABCG1 and ABCA1 are responsible for the majority of cholesterol efflux from primary macrophages to mouse whole serum. A very similar estimate of the combined contribution of ABCA1 and ABCG1 to mass cholesterol efflux to HDL was obtained by knocking down ABCA1 in ABCG1/- primary peritoneal macrophages (22).

We performed in vivo RCT experiments using BMMs from ABCG1-KO mice. The plasma $\left[{ }^{3} \mathrm{H}\right]$ cholesterol levels in mice injected with ABCG1-KO BMMs were decreased by $20 \%(P<0.001)$, as measured by the area under the curve, compared with those in mice injected with WT BMMs (Figure 5C). Total fecal $\left[{ }^{3} \mathrm{H}\right]$ steroid excretion was $25 \%$ lower in feces collected from ABCG1-KO BMM-injected mice $(P<0.01$; Figure 5D). Similar results were obtained in a second independent RCT experiment using BMMs. Furthermore, 2 separate RCT experiments using MPMs from ABCG1deficient and control mice resulted in an average decrease of $25 \%(P<0.05)$ in plasma $\left[{ }^{3} \mathrm{H}\right]$ tracer level, as calculated by the area under curve, and average decrease of $27 \%(P<0.05)$ in fecal $\left[{ }^{3} \mathrm{H}\right]$ tracer excretion (Supplemental Figure 3, A and B). Thus, primary macrophages that lack ABCG1 expression had significantly impaired macrophage RCT in vivo.

$A B C A 1$ and $A B C G 1$ cooperatively mediate macrophage RCT in vivo. To investigate whether ABCA1 and ABCG1 function cooperatively in mediating macrophage RCT in vivo, we generated stable ABCA1and ABCG1-double-KD (ABCG1-DKD) J774 cells by transfecting and stably expressing shRNA against ABCA1 in the ABCG1-KD cells described above. The abca1 mRNA level was reduced by $50 \%$, while abcg1 mRNA levels remained unchanged in DKD cells compared with ABCG1KD cells (Figure 6A). While ABCG1-KD cells had no significant decrease in cholesterol efflux to free apoA-I, the DKD cells had a greater than $85 \%$ reduction in cholesterol efflux to free apoA-I (Figure 6B), indicating that ABCA1 was effectively knocked down in these cells. When mouse whole serum was used as the acceptor, cholesterol efflux from ABCG1-KD cells was reduced by $30 \%$ (Figure 6C), while DKD cells had a 10\% further decrease in cholesterol efflux to mouse serum compared with ABCG1-KD cells (Figure 6C). Probucol treatment (to inhibit ABCA1 function) reduced cholesterol efflux to mouse serum by only an additional $14 \%$ in the DKD cells (Figure 6C). These data suggest that ABCA1 and ABCG1 cooperatively mediate cholesterol efflux to mouse serum.

We performed in vivo RCT experiments using control, ABCG1$\mathrm{KD}$, and DKD cells. As above, mice injected with ABCG1-KD cells had significantly reduced plasma and fecal $\left[{ }^{3} \mathrm{H}\right]$ tracer levels com- 
A
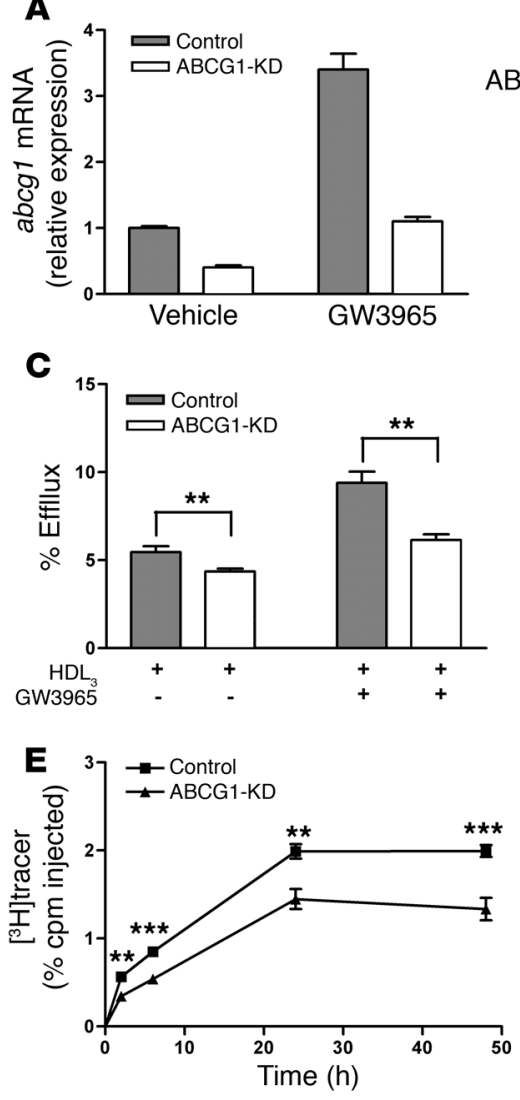

B
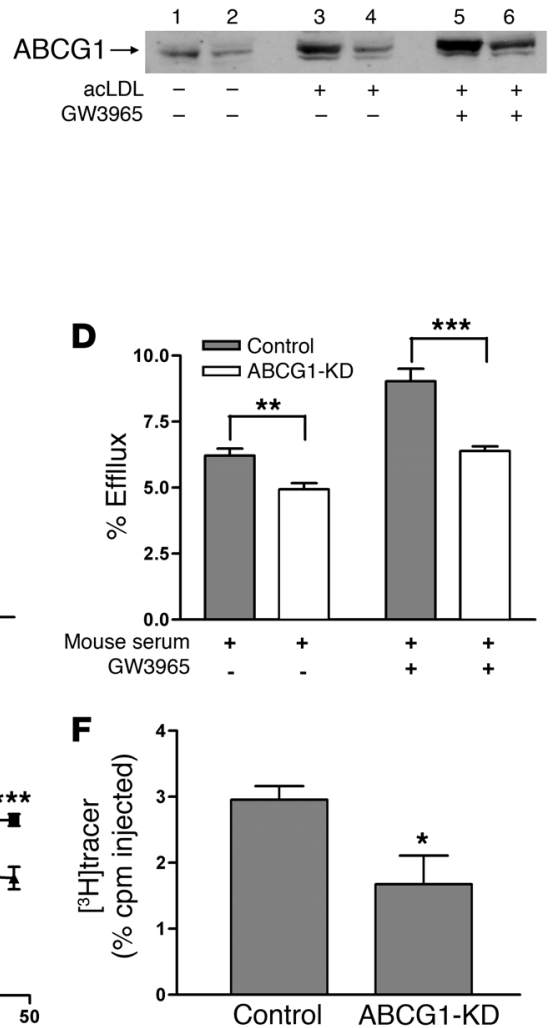

\section{Figure 4}

Knockdown of ABCG1 in $\mathrm{J} 774$ macrophages reduces cholesterol efflux in vitro and RCT in vivo. (A) Quantitative analysis of mRNA expression of abcg1 in ABCG1-KD and control cells by quantitative RT-PCR. Total RNA was extracted from cells that were treated with either vehicle or $1 \mu \mathrm{M}$ GW3965 for 24 hours. Data are expressed as fold change \pm SD and normalized to mouse 18S rRNA. (B) Western blotting demonstrating the knockdown of ABCG1 protein expression. Control and $A B C G 1-K D$ cells were treated with either acLDL or GW3965. ABCG1 was detected by Western blotting with anti-ABCG1 antibody. Equal amount of total proteins were loaded. Lanes 1, 3, and 5 represent control cells. Lanes 2, 4, and 6 represent ABCG1-KD cells. (C and D) Cholesterol efflux assay was performed as described in Figure 1 in the presence of $\mathrm{HDL}_{3}(25 \mu \mathrm{g} / \mathrm{ml})$ or $2.5 \%$ mouse whole serum for 4 hours. Data are expressed as mean $\pm \mathrm{SD} ; n=3$. ${ }^{\star \star} P<0.01 ;{ }^{* \star} P<0.001$. (E and F) The RCT experiment was performed as described in Figure 1 with $\left[{ }^{3} \mathrm{H}\right]$ cholesterol-labeled, acLDL-loaded, and LXR agonist-treated J774 control macrophages and ABCG1-KD macrophages. $n=8$ mice per group. Data are expressed as the percentage of tracer relative to total cpm tracer injected \pm SEM. ${ }^{*} P<0.05$; ${ }^{* \star} P<0.01$; ${ }^{* * *} P<0.001$. Results are representative of 3 independent experiments. (E) Time course of $\left[{ }^{3} \mathrm{H}\right]$ cholesterol distribution in plasma. Individual time points and areas under the curve were determined and compared. (F) Fecal $\left[{ }^{3} \mathrm{H}\right]$ tracer levels. Feces were collected continuously from 0 to 48 hours. pared with mice injected with control cells (Figure 6, D and E). More importantly, the plasma $\left[{ }^{3} \mathrm{H}\right]$ cholesterol level in mice injected with DKD cells was decreased by $15 \%(P<0.01)$ compared with that in mice injected with ABCG1-KD cells and by $66 \%(P<0.001)$ compared with that in mice injected with control cells, as calculated by the area under curve (Figure 6D). The total fecal $\left[{ }^{3} \mathrm{H}\right]$ steroid excretion was also reduced by $23 \%(P<0.05)$ compared with that in mice injected with ABCG1-KD cells and $40 \%(P<0.001)$ compared with that in mice injected with control cells (Figure 6E). Taken together, these data indicate that ABCG1 and ABCA1 contribute in an additive fashion to macrophage RCT in vivo.

\section{Discussion}

In the present study, we show quantitatively that macrophage ABCA1 contributes to macrophage RCT in vivo, but that substantial residual efflux and RCT still occur. Mice that were injected with macrophages lacking ABCA1 expression had significantly lower macrophage-derived $\left[{ }^{3} \mathrm{H}\right]$ cholesterol levels in plasma and feces over the 48-hour experimental period compared with mice injected with WT macrophages, but there was still a substantial amount of tracer appearing in plasma and being excreted in feces even in mice injected with $\mathrm{ABCA} 1^{-/-}$macrophages. We investigated whether macrophage SR-BI expression might mediate this residual efflux and showed that macrophage RCT was not significantly different in mice injected with either SR-BI-/- or control macrophages, suggesting that macrophage SR-BI does not substantially contribute to macrophage RCT in vivo. Next, we determined whether macrophage ABCG1 was able to promote macrophage cholesterol efflux and RCT in vivo. We utilized stable ABCG1-OE macrophages to show that elevated ABCG1 expression promoted cholesterol efflux and RCT in vivo. Conversely, we showed that stable ABCG1-KD macrophages or primary ABCG1-KO macrophages reduced cholesterol efflux and RCT in vivo. Finally, we knocked down both ABCA1 and ABCG1 and demonstrated that these DKD macrophages had significantly reduced RCT in vivo compared with single ABCG1-KD cells. Thus, both ABCA1 and ABCG1, but not SR-BI, are key regulators of macrophage RCT in vivo.

Previously, in vitro data have shown that ABCA1 plays important roles in cholesterol and phospholipid efflux to lipid-poor apoA-I but not to mature HDL. Mutations in $A B C A 1$ cause Tangier disease in patients that is characterized by HDL deficiency and increased susceptibility to atherosclerosis (23-26). Specific deletion of ABCA1 expression in macrophages in LDL receptor-KO (LDLR$\mathrm{KO}$ ) mice induced atherosclerosis (7). Conversely, overexpression of ABCA1 in macrophages inhibited atherosclerotic lesion development in LDLR-KO mice (8). No significant changes in plasma lipid levels were observed in either study. These data suggest that macrophage ABCA1 plays a role in preventing atherosclerosis, independent of plasma HDL cholesterol levels. Our results here are consistent with the concept that macrophage ABCA1 protects against atherosclerosis at least in part by promoting macrophage cholesterol efflux and RCT in vivo.

Importantly, there was substantial residual RCT even from ABCA1-deficient macrophages, suggesting the existence of alternative pathways that are relevant in vivo. SR-BI is known to promote cholesterol efflux from macrophage to HDL as an acceptor (27), but this flux can be bidirectional and has uncertain effects on macrophage cholesterol mass. Hepatic SR-BI is certainly a critical regu- 

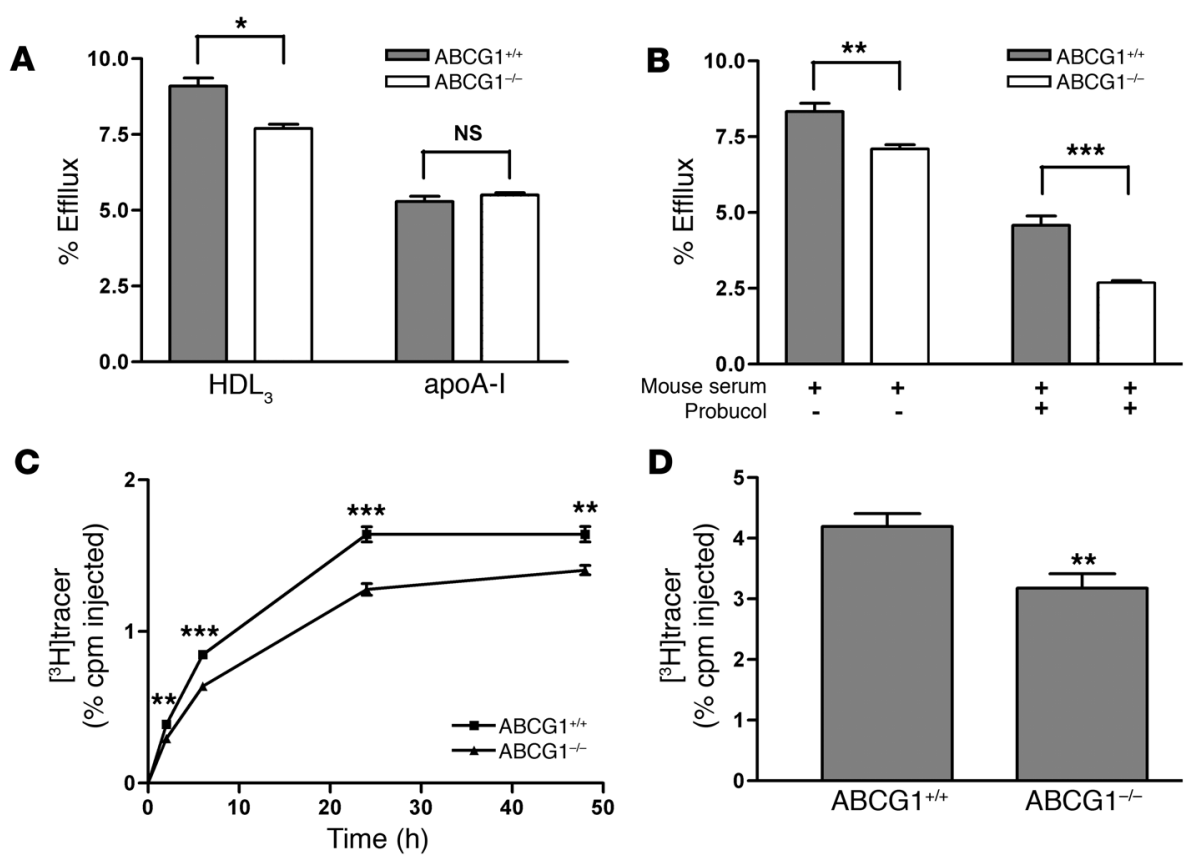

\section{Figure 5}

ABCG1-deficient BMMs have reduced cholesterol efflux ex vivo and RCT in vivo. (A) Cholesterol efflux assay was determined as described in Figure 1 with BMMs from WT (ABCG1 ${ }^{+/+}$) or ABCG1-KO $\left(A B C G 1^{-1}\right)$ mice. Cholesterol efflux was determined in the presence of $\mathrm{HDL}_{3}(25 \mu \mathrm{g} / \mathrm{ml})$ or lipid-free apoA-I $(10 \mu \mathrm{g} / \mathrm{ml})$ for 2 hours. Data are expressed as mean $\pm \mathrm{SD} ; n=3$. ${ }^{*} P<0.05$. (B) Cholesterol efflux was determined as described above, except cells were incubated for 2 hours either in the presence or absence probucol $(20 \mu \mathrm{M})$ prior to the addition of $2.5 \%$ mouse whole serum as the acceptor. Data are expressed as mean $\pm \mathrm{SD} ; n=3 .{ }^{* \star} P<0.01 ;{ }^{* * *} P<0.001$. (C and D) The RCT assay was performed as described in Figure 1 with $\left[{ }^{3} \mathrm{H}\right]$ cholesterol-labeled, acLDL-loaded, and LXR agonisttreated $\mathrm{BMMs}$ from $\mathrm{ABCG} 1^{+/+}$or $\mathrm{ABCG} 1^{-/-}$mice. $n=8$ mice per group. Data are expressed as the percentage of tracer relative to total $\mathrm{cpm}$ tracer injected \pm SEM. ${ }^{* \star} P<0.01 ;{ }^{* \star \star} P<0.001$. Results are representative of 2 independent experiments. (C) Time course of $\left[{ }^{3} \mathrm{H}\right]$ cholesterol distribution in plasma. Individual time points and areas under the curve were determined and compared. (D) Fecal $\left[{ }^{3} \mathrm{H}\right]$ tracer levels. Feces were collected continuously from 0 to 48 hours.

lator of HDL metabolism in mice, as SR-BI KO mice have elevated large, cholesterol-rich HDL in plasma (28). SR-BI-KO mice have markedly accelerated atherosclerosis $(11,28,29)$, related at least in part to impaired RCT due to hepatic deficiency of SR-BI (10). Mice transplanted with BM from SR-BI-deleted mice also generally develop increased atherosclerosis $(11,12,30)$, but the mechanism of this effect is unknown and not proven to be impaired macrophage $\mathrm{RCT}$. We found that $\mathrm{SR}-\mathrm{BI}^{-/-}$macrophages had similar levels of cholesterol efflux to $\mathrm{HDL}_{3}$ ex vivo, and, more importantly, when we injected SR-BI-/- and control primary macrophages into mice, similar levels of RCT were observed, suggesting that macrophage SR-BI does not contribute to macrophage RCT in vivo. Our data here suggest that the mechanisms by which hematopoetically derived SR-BI is atheroprotective are likely to be independent of SR-BI-mediated cholesterol efflux from macrophages.

We further investigated the quantitative role of ABCG1 in macrophage cholesterol efflux and RCT in vivo. ABCA1 and ABCG1 are regulated by LXR in a similar fashion. Macrophages that are loaded with acLDL express significantly higher levels of both ABCA1 and ABCG1, a mechanism that protects against cholesterol toxicity by promoting free cholesterol efflux. Previous studies have shown that $\mathrm{ABCG} 1$, in contrast to $\mathrm{ABCA} 1$, promotes choles- terol efflux from macrophages to HDL particles but not to lipid-poor apoA-I in vitro $(13,14)$. Furthermore, mice deficient in ABCG1 have increased tissue macrophage lipid accumulation in vivo $(14,18)$. Our studies here used 3 independent approaches to assess specifically whether macrophage ABCG1 is involved in macrophage cholesterol efflux and RCT in vivo. First, J774 macrophages overexpressing ABCG1 had significantly increased RCT in vivo compared with control macrophages. Second, J774 macrophages with reduced ABCG1 expression demonstrated impaired RCT in vivo. Third, primary macrophages (both BMMs and MPMs) with ablated ABCG1 expression also had significantly decreased RCT in vivo. These data demonstrate that ABCG1 promotes macrophage cholesterol efflux and RCT in vivo.

Our results suggest that macrophage ABCG1 might be expected to be inhibitory of atherosclerosis development by promoting macrophage efflux and RCT in vivo. However, several recent studies have provided conflicting results on the roles of macrophage ABCG1 in the development of atherosclerosis in mice. Out et al. showed that macrophage deletion of $A b c g 1$ led to a modest but significant increase in atherosclerotic lesion development in LDLR-KO mice (19). However, 2 other studies observed decreased atherosclerosis in $\mathrm{LDLR}^{-/-}$mice transplanted with $A b c g 1^{-/-} \mathrm{BM}(17,18)$. Each group has provided different mechanistic explanations that are not mutually exclusive. Ranalletta et al. (17) observed significant induction of ABCA1 and increased apoE secretion in ABCG1-deficient macrophages and suggested that these compensated for the lack of ABCG1-mediated cholesterol efflux. Baldan et al. (18) observed accelerated apoptosis of $\mathrm{Abcg} 1^{-/-}$ macrophages and suggested that this might result in smaller atherosclerotic lesions. Clearly, macrophage cholesterol efflux and RCT constitute only one factor involved in the overall impact of an $\mathrm{ABC}$ transporter on atherosclerosis, and other factors, such as compensatory upregulation of LXR target genes and apoptosis, can also have effects on atherosclerosis beyond those of RCT.

ABCA1 and ABCG1 have been shown to mediate cholesterol efflux to lipid-poor apoA-I and mature HDL, respectively. Recent studies indicate that ABCA1 may lipidate lipid-poor apoA-I to generate nascent HDL, which can then act as acceptor for ABCG1mediated cholesterol efflux (15). It is not known whether ABCA1 and ABCG1 function dependently or independently in the process of macrophage cholesterol efflux and RCT in vivo. To further investigate the role of ABCA1 and ABCG1 in macrophage RCT in vivo, we generated DKD J774 cells. Cholesterol efflux to free apoA-I was reduced by more than $85 \%$ from the DKD cells, suggesting that ABCA1 expression was very effectively suppressed. Mice 
A

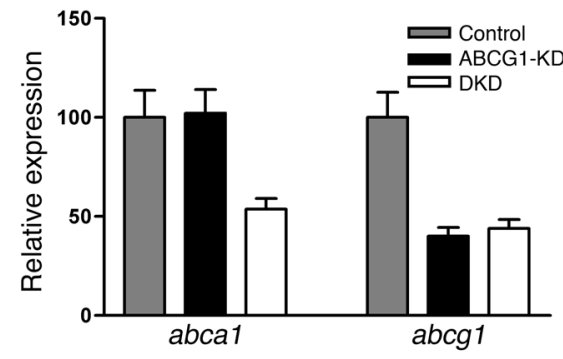

C
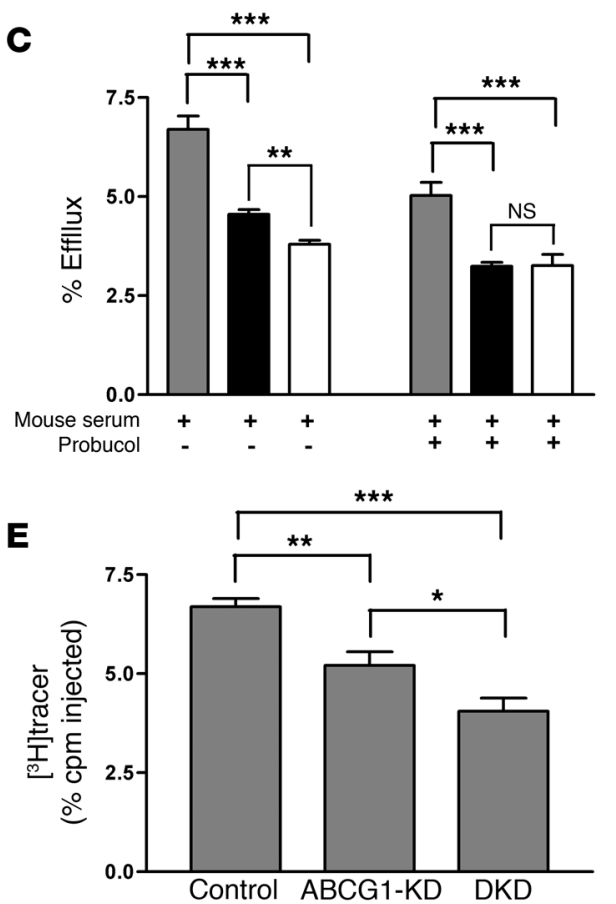

B

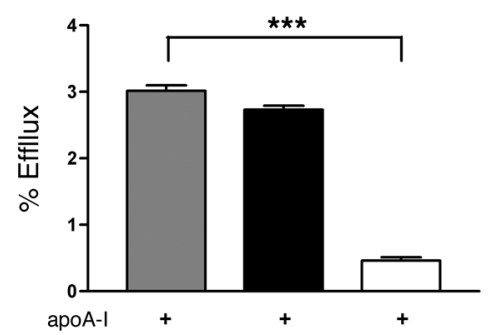

D

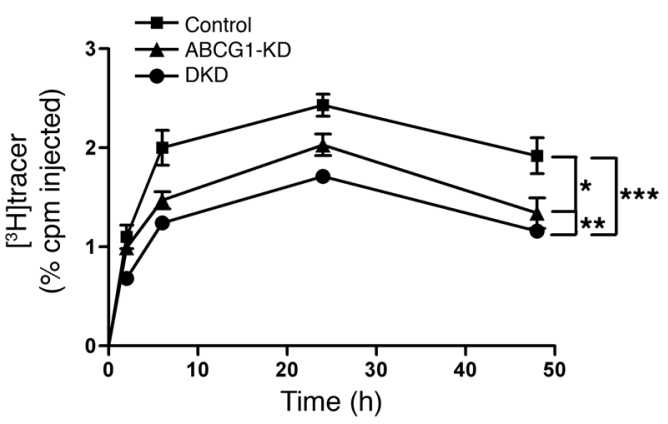

In summary, we demonstrate that macrophage ABCA1 and ABCG1, but not SR-BI, promote RCT in vivo and that $\mathrm{ABCA} 1$ and $\mathrm{ABCG} 1$ are additive in their effects. These results suggest that therapeutic intervention to increase macrophage $\mathrm{ABCA} 1$ and $A B C G 1$ expression may be an effective strategy to enhance macrophage RCT and potentially reduce atherosclerosis.

\section{Methods}

Reagents and plasmid constructs. Blasticidin S $\mathrm{HCl}$ and G418 were purchased from Invitrogen. The LXR agonist GW3965 was obtained from GlaxoSmithKline and has been described previously (31). The shRNA sequences for murine ABCG1 were designed using Invitrogen's online BLOCK-iT RNAi Designer (www. invitrogen.com/rnai). Control shRNA sequences for $l a c Z$ were provided by Invitrogen. Complementary DNA oligonucleotides were annealed and cloned into pENTR/U6 (Invitrogen). Then the expression constructs were generated by performing an LR recombination reaction between the pENTR/U6 entry construct and pLenti6/BLOCK-iT-DEST expression construct according to the manufacturer's instructions. Final constructs were confirmed by DNA sequencing. The murine Abcg1 RNAi sequence was: 5'-GGAAGTCCACACTCATGAATA-3'. SureSilencing shRNA and scrambled control plasmids for murine ABCA1 were purchased from SuperArray Bioscience Corp. The murine Abca1 RNAi sequence was: $5^{\prime}$-TCCCTGGGTGTCAGTAATTCT-3'. injected with DKD cells had a modest but significant decrease in macrophage RCT compared with mice injected with ABCG1-KD cells. These data suggest that ABCA1 and ABCG1 cooperatively contribute to macrophage RCT in vivo.

There was still a substantial level of RCT remaining in mice injected with ABCA1 and ABCG1 DKD cells. This is likely due in part to the residual expression of ABCA1 and ABCG1 in these J774$\mathrm{KD}$ cells. In addition, passive diffusion could account for some of the ABCA1/ABCG1-independent cholesterol efflux out of macrophages. Finally, it is possible that there are additional molecular regulators of macrophage efflux that are yet to be identified.
Lentiviral vector transduction. Lentiviral stocks were produced by cotransfecting the pLenti6/BLOCK-iT-DEST constructs expressing shRNA targeting lacZ and Abcg1 with ViraPower Packaging Mix (Invitrogen) into the 293FT Producer Cell Line (Invitrogen). Then, these lentiviral stocks were used to transduce WT J774 cells. Stably transduced cell lines were selected in DMEM supplemented with $10 \% \mathrm{FBS}$ and $5 \mu \mathrm{g} / \mathrm{ml}$ blasticidin. RNA expression of targeted gene in each cell line was examined by quantitative RT-PCR.

Cell culture. J774 cells obtained from ATCC were grown in DMEM supplemented with $10 \%$ FBS. J774-control (stably expressing shRNA targeting lacZ) and J774-ABCG1-KD (stably expressing shRNA targeting murine Abcg1) were grown in DMEM supplemented with $10 \%$ FBS and $5 \mu \mathrm{g} / \mathrm{ml}$ blasticidin. 
J774-DKD (stably expressing shRNAs targeting murine Abcg1 and Abca1) was grown in DMEM supplemented with $10 \% \mathrm{FBS}$ and $500 \mu \mathrm{g} / \mathrm{ml}$ G418. J774-ABCG1-OE (stably overexpressing murine ABCG1) was grown in DMEM supplemented with 10\% FBS. For the RCT experiment, J774 cells were grown as described previously, with the exception that the medium was supplemented with $2.5 \mu \mathrm{g} / \mathrm{ml}$ blasticidin (31). BMMs were isolated from femurs and tibias and cultured in DMEM supplemented with $10 \%$ FBS and 30\% L929 conditioned medium (32). MPMs were isolated from mice 3 days after i.p. injection of $1 \mathrm{ml}$ of $3.8 \%$ thioglycollate medium. For the RCT experiment, both BMMs and MPMs were grown in 100-mm Petri dishes, labeled with $5 \mu \mathrm{Ci} / \mathrm{ml}\left[{ }^{3} \mathrm{H}\right]$ cholesterol, and loaded with $25 \mu \mathrm{g} / \mathrm{ml}$ acLDL. Twenty-four hours later, cells were washed and equilibrated overnight in DMEM-BSA (0.2\%). Before the injection, cells were collected in PBS-EDTA $(10 \mathrm{mM})$, washed, and suspended in MEM/HEPES.

Animals. WT C57BL/6 and DBA/J mice were obtained from The Jackson Laboratory. Abca1-and Srb1-KO mice were originally purchased from The Jackson Laboratory and subsequently bred in-house. Abcg1-KO mice were purchased from Deltagen and then backcrossed 5 generations into the WT C57BL/ 6 background. Mice were fed a standard chow diet ad libitum. Eight- to 12 -week-old female C57BL/6 mice were fed a diet containing 120 ppm GW3965 (Research Diets Inc.) for 2 weeks before and during the course of the experiment. Plasma lipid levels were determined before and after mice were put on the GW3965 diet. Cell injection and blood and feces collection were performed as described previously $(31,33)$. All animal experiments were approved by the Institutional Animal Care and Use Committee of the University of Pennsylvania.

RNA isolation and gene expression analysis. Total RNA was isolated using EZ1 RNA Mini Kit (QIAGEN) according to the manufacturer's instructions. Then $0.5 \mu \mathrm{g}$ of total RNA was reverse transcribed using QuantiTect Reverse Transcription Kit (QIAGEN). Real-time quantitative PCR was performed on an Applied Biosystems 7300 sequence detector. Primer and probe sequences were as described previously (31).

Cholesterol efflux assays. Cells were labeled with $\left[{ }^{3} \mathrm{H}\right]$ cholesterol $(2 \mu \mathrm{Ci} / \mathrm{ml}$; Amersham) and loaded with $25 \mu \mathrm{g} / \mathrm{ml}$ acLDL for 24 hours. Then cells were washed and equilibrated overnight in either the presence or absence of GW3965 (1 $\mu \mathrm{M})$ in serum-free medium. For the cholesterol efflux, medium containing $25 \mu \mathrm{g} / \mathrm{ml} \mathrm{HDL}, 10 \mu \mathrm{g} / \mathrm{ml}$ free apoA-I, or $2.5 \%$ mouse serum was added to cells. After 2 or 4 hours, aliquots of the medium were removed, and the $\left[{ }^{3} \mathrm{H}\right]$ cholesterol released was measured by liquid scintillation counting. The $\left[{ }^{3} \mathrm{H}\right]$ cholesterol present in the cells was determined by extracting the cell lipids in isopropanol and measured by liquid scintillation counting. Cells were treated with $10 \mu \mathrm{M}$ probucol for 2 hours (where indicated) before acceptors were added to cells (34).

Western blotting. Total cell lysis was prepared using M-PER reagent (Pierce) according to the manufacturer's instructions. Equal amount of total proteins were loaded on NuPAGE Mini-gel (Invitrogen). Primary antibodies for ABCG1 were purchased from Santa Cruz Biotechnology Inc. Primary antibody for SR-BI was purchased from Novus Biologicals.

Statistics. Area under the curve and statistical analysis were determined by 2-tailed Student's $t$ test and ANOVA with the use of GraphPad Prism Software version 3.0. Results are presented as mean \pm SD or \pm SEM. Statistical significance was reached when $P<0.05$.

\section{Acknowledgments}

This work was supported by P01-HL22633 (to G.H. Rothblat and D.J. Rader), R01-HL55323 (to D.J. Rader), and R01-HL54591 (to A.R. Tall) from the National Heart, Lung, and Blood Institute and an Alternative Drug Discovery Initiative award to the University of Pennsylvania from GlaxoSmithKline. We thank Colin MacPhee and Michael Jaye for helpful discussions and for providing the LXR agonist GW3965. We are indebted to Dawn Marchadier, Aisha Wilson, Edwige Edouard, Michelle Joshi, Anna DiFlorio, and Linda Morrell for their excellent technical assistance.

Received for publication March 8, 2007, and accepted in revised form May 10, 2007.

Address correspondence to: Daniel J. Rader, University of Pennsylvania School of Medicine, Center for Experimental Therapeutics, 421 Curie Boulevard, 654 BRBII/III Labs, Philadelphia, Pennsylvania 19104-6160, USA. Phone: (215) 573-4176; Fax: (215) 573-8606; E-mail: rader@mail.med.upenn.edu.
1. Cuchel, M., and Rader, D.J. 2006. Macrophage reverse cholesterol transport: key to the regression of atherosclerosis? Circulation. 113:2548-2555.

2. Linsel-Nitschke, P., and Tall, A.R. 2005. HDL as a target in the treatment of atherosclerotic cardiovascular disease. Nat. Rev. Drug Discov. 4:193-205.

3. Wang, N., Silver, D.L., Costet, P., and Tall, A.R. 2000. Specific binding of ApoA-I, enhanced cholesterol efflux, and altered plasma membrane morphology in cells expressing ABC1. J. Biol. Chem. 275:33053-33058

4. Oram, J.F., Lawn, R.M., Garvin, M.R., and Wade, D.P. 2000. ABCA1 is the cAMP-inducible apolipoprotein receptor that mediates cholesterol secretion from macrophages. J. Biol. Chem. 275:34508-34511.

5. Bortnick, A.E., et al. 2000. The correlation of ATP-binding cassette $1 \mathrm{mRNA}$ levels with cholesterol efflux from various cell lines. J. Biol. Chem. 275:28634-28640.

6. Haghpassand, M., Bourassa, P.-A.K., Francone, O.L., and Aiello, R.J. 2001. Monocyte/macrophage expression of ABCA1 has minimal contribution to plasma HDL levels. J. Clin. Invest. 108:1315-1320. doi:10.1172/JCI200112810.

7. Van Eck, M., et al. 2002. Leukocyte ABCA1 controls susceptibility to atherosclerosis and macrophage recruitment into tissues. Proc. Natl. Acad. Sci. U. S. A. 99:6298-6303.

8. Van Eck, M., et al. 2006. Macrophage ATP-bind- ing cassette transporter $\mathrm{A} 1$ overexpression inhibits atherosclerotic lesion progression in low-density lipoprotein receptor knockout mice. Arterioscler. Thromb. Vasc. Biol. 26:929-934.

9. Yancey, P.G., et al. 2000. High density lipoprotein phospholipid composition is a major determinant of the bi-directional flux and net movement of cellular free cholesterol mediated by scavenger receptor BI. J. Biol. Chem. 275:36596-36604.

10. Zhang, Y., et al. 2005. Hepatic expression of scavenger receptor class B type I (SR-BI) is a positive regulator of macrophage reverse cholesterol transport in vivo. J. Clin. Invest. 115:2870-2874. doi:10.1172/ JCI25327.

11. Covey, S.D., Krieger, M., Wang, W., Penman, M., and Trigatti, B.L. 2003. Scavenger receptor class B type Imediated protection against atherosclerosis in LDL receptor-negative mice involves its expression in bone marrow-derived cells. Arterioscler. Thromb. Vasc. Biol. 23:1589-1594.

12. Zhang, W., et al. 2003. Inactivation of macrophage scavenger receptor class B type I promotes atherosclerotic lesion development in apolipoprotein E-deficient mice. Circulation. 108:2258-2263.

13. Wang, N., Lan, D., Chen, W., Matsuura, F., and Tall, A.R. 2004. ATP-binding cassette transporters G1 and G4 mediate cellular cholesterol efflux to highdensity lipoproteins. Proc. Natl. Acad. Sci. U. S. A. 101:9774-9779.

14. Kennedy, M.A., et al. 2005. ABCG1 has a critical role in mediating cholesterol efflux to HDL and preventing cellular lipid accumulation. Cell Metab. 1:121-131.

15. Gelissen, I.C., et al. 2006. ABCA1 and ABCG1 synergize to mediate cholesterol export to apoA-I. Arterioscler. Thromb. Vasc. Biol. 26:534-540.

16. Baldan, A., et al. 2006. Deletion of the transmembrane transporter ABCG1 results in progressive pulmonary lipidosis. J. Biol. Chem. 281:29401-29410.

17. Ranalletta, M., et al. 2006. Decreased atherosclerosis in low-density lipoprotein receptor knockout mice transplanted with Abcg 1/-- bone marrow. Arterioscler. Thromb. Vasc. Biol. 26:2308-2315.

18. Baldan, A., et al. 2006. Impaired development of atherosclerosis in hyperlipidemic $\mathrm{Ldlr}^{-/-}$and $\mathrm{ApoE}^{-/-}$ mice transplanted with Abcg 1/-- bone marrow. Arterioscler. Thromb. Vasc. Biol. 26:2301-2307.

19. Out, R., et al. 2006. Macrophage ABCG1 deletion disrupts lipid homeostasis in alveolar macrophages and moderately influences atherosclerotic lesion development in LDL receptor-deficient mice. Arterioscler. Thromb. Vasc. Biol. 26:2295-2300.

20. Yu, L., Cao, G., Repa, J., and Stangl, H. 2004. Sterol regulation of scavenger receptor class B type I in macrophages. J. Lipid Res. 45:889-899.

21. Tontonoz, P., and Mangelsdorf, D.J. 2003. Liver X receptor signaling pathways in cardiovascular disease. Mol. Endocrinol. 17:985-993.

22. Yvan-Charvet, L., et al. 2007. Inhibition of cholesteryl ester transfer protein by torcetrapib modestly 
increases macrophage cholesterol efflux to HDL. Arterioscler. Thromb. Vasc. Biol. 27:1132-1138.

23. Bodzioch, M., et al. 1999. The gene encoding ATPbinding cassette transporter 1 is mutated in Tangier disease. Nat. Genet. 22:347-351.

24. Brooks-Wilson, A., et al. 1999. Mutations in ABC1 in Tangier disease and familial high-density lipoprotein deficiency. Nat. Genet. 22:336-345.

25. Remaley, A.T., et al. 1999. Human ATP-binding cassette transporter 1 (ABC1): genomic organization and identification of the genetic defect in the original Tangier disease kindred. Proc. Natl. Acad. Sci. U. S. A. 96:12685-12690.

26. Rust, S., et al. 1999. Tangier disease is caused by mutations in the gene encoding ATP-binding cassette transporter 1. Nat. Genet. 22:352-355.
27. Rothblat, G.H., et al. 1999. Cell cholesterol efflux: integration of old and new observations provides new insights. J. Lipid Res. 40:781-796.

28. Trigatti, B., et al. 1999. Influence of the high density lipoprotein receptor SR-BI on reproductive and cardiovascular pathophysiology. Proc. Natl. Acad. Sci.U. S. A. 96:9322-9327.

29. Braun, A., et al. 2002. Loss of SR-BI expression leads to the early onset of occlusive atherosclerotic coronary artery disease, spontaneous myocardial infarctions, severe cardiac dysfunction, and premature death in apolipoprotein E-deficient mice. Circ. Res. 90:270-276.

30. Van Eck, M., Bos, I.S., Hildebrand, R.B., Van Rij, B.T., and Van Berkel, T.J. 2004. Dual role for scavenger receptor class B, type I on bone marrow-derived cells in atherosclerotic lesion development. Am.J. Pathol. 165:785-794.

31. Naik, S.U., et al. 2006. Pharmacological activation of liver X receptors promotes reverse cholesterol transport in vivo. Circulation. 113:90-97.

32. Schiller, N.K., Black, A.S., Bradshaw, G.P., Bonnet, D.J., and Curtiss, L.K. 2004. Participation of macrophages in atherosclerotic lesion morphology in LDLr $^{-/}$mice. J. Lipid Res. 45:1398-1409.

33. Zhang, Y., et al. 2003. Overexpression of apolipoprotein A-I promotes reverse transport of cholesterol from macrophages to feces in vivo. Circulation. 108:661-663.

34. Favari, E., et al. 2004. Probucol inhibits ABCA1mediated cellular lipid efflux. Arterioscler. Thromb. Vasc. Biol. 24:2345-2350. 